\title{
Method for Image Source Separation by Means of Independent Component Analysis: ICA, Maximum Entory Method: MEM, and Wavelet Based Method: WBM
}

\author{
Kohei Arai ${ }^{1}$ \\ Graduate School of Science and Engineering \\ Saga University \\ Saga City, Japan
}

\begin{abstract}
Method for image source separation based on Independent Component Analysis: ICA, Maximum Entropy Method: MEM, and Wavelet Based Method: WBM is proposed. Experimental results show that image separation can be done from the combined different images by using the proposed method with an acceptable residual error.
\end{abstract}

Keywords-Blind separation; image separation; cucktail party effect; ICA; MEM; wavelet analuysis.

\section{INTRODUCTION}

There are some technologies and systems which allow separate the specific speaker from the mixed image data which is acquired at some noisy circumstances. The related application studies are conducted, in particular, for TV meeting environment, image recognition systems, and digital hearing aid system etc. In particular, the microphone array system as well as Independent Component Analysis (ICA) base approach [1] is focused. Microphone array allows enhance a target image from the mixed images suppressing noises and taking into account the phase difference among the imagesources which corresponds to the distance between the microphone and the location of the imagesources. There is a delay sum [2] and an adaptation [3] types array microphone systems. These types of array microphone allow direct the beam to the desired direction of the target of interest.

ICA is the method which allows the blind separation based on the imagesources are isolated each other. ICA based method configures reconstruction filter maximizing KullbackLeibler Divergence [4] for separation of target imagesources in concern from the acquired images [5], [6].

The blind separation method with entropy maximization rule is proposed [7]. In order to improve separability among the possible imagesources, high frequency component derived from the wavelet based Multi Resolution Analysis (MRA) [8],[9] is used in the entropy maximization rule utilized method. Due to the fact that the MRA based separability improvement is not good enough, further improvement is required.
Blind separation method which is proposed here is based on the MRA based separability improvement. A single level of MRA is not good enough for characteristics enhancement of each imagesource. Therefore, the level is considered as a parameter for MRA utilized blind separation [10]. An appropriate level is found for improvement of separability then blind separation is applied. It is found that the proposed method can achieve 4 to $8.8 \%$ of separability improvement for the case of the number of speakers is 2, 4 and 8 [11].

The following section describes the proposed method followed by some experiments with the mixed images. Then conclusions and some discussions is followed.

\section{PRoposed MTHEOD}

\section{A. Image Mikxing Model}

Assuming the original image, $x_{i}(t)$ is mixed with several images, for instance $s_{1}(t)$ and $s_{2}(t)$ as shown in equation (1).

$x_{i}(t)=a_{i 1} s_{1}(t)+a_{i 2} s_{2}(t)$

where $a_{i 1}$ and $a_{i 2}$ are weighting factor, mixing ratio. Also it is assumed that $s_{1}(t)$ and $s_{2}(t)$ are mutually independent. Although this is an example of image mixing model for just two images, it is possible to expand the number of images which are to be mixed together.

\section{B. Maximum Entropy Method}

Maximum Entropy Method: MEM is used for learning processes for maximizing combined entropy in two layered neural network

$$
\begin{aligned}
& y_{i}=g\left(\sum_{k=1}^{2} w_{i k} x_{k}-\theta_{i}\right) \\
& g(v)=1-e^{-v} / 1+e^{-v}
\end{aligned}
$$

where $x, y$ notes input and output signals, or images while $w$ denotes weighting coefficients of two layered neural network. $\Theta$ denotes a threshold. $g(v)$ is called sigmoid function. (4). 


$$
H(y)=\langle\ln (|J|)\rangle-\langle\ln (p(x))\rangle
$$

where $J$ denotes Jacobian matrix as shown in equation (5)

$J=\operatorname{det}\left(\begin{array}{ll}\partial y_{1} / \partial x_{1} & \partial y_{1} / \partial x_{2} \\ \partial y_{2} / \partial x_{1} & \partial y_{2} / \partial x_{2}\end{array}\right)$

Because the second term of the equation (4) is constant, the following equation (6) has to be maximized.

$$
I=-\langle\ln (|J|)\rangle
$$

\section{Steepest Descending Method}

In order to maximize the equation (6), Steepest Descending Method: SDM is used. Updating equations for $\mathrm{w}$ and $\theta$ can be expressed with equations (7) and (8).

$$
\begin{aligned}
& w_{i k}^{\text {new }}=w_{i k}^{\text {old }}-\mathrm{\gamma} \partial I / \partial w_{i k} \\
& \theta_{i}^{\text {new }}=\theta_{i}^{\text {old }}-\mathrm{\gamma} \partial I / \partial \theta_{i}
\end{aligned}
$$

More precisely, updating equations can be re-written by equation (9) and (10).

$$
\begin{aligned}
& W^{\text {new }}=W^{\text {old }}+\mathrm{y}\left\langle\left[W^{T}\right]^{-1}+2 \mathrm{yx}^{T}\right\rangle \\
& \theta^{\text {new }}=\theta^{\text {old }}+\mathrm{y}\langle 2 \mathrm{y}\rangle
\end{aligned}
$$

Thus the original images are separated from the combined image with the proposed method.

\section{Discrete Wavelet Transformation}

In order to separate several original images from the given combined images, Discrete Wavelet Transformation: DWT is used. DWT allows decompose images with high and low wavelet frequency components orthogonally. There are many orthogonal base functions for DWT. One of the based functions is Haar function. Figure 1 shows an example of the original image of Lena in the SIDBA standard image database, and decomposed image through WT. In the decomposed image, LL image, low frequency component in horizontal direction and low frequency component in vertical direction is situated at the top left corner while $\mathrm{LH}, \mathrm{HL}$, and $\mathrm{HH}$ components are situated at the top right, the bottom left, and the bottom right corners, respectively.

Histogram of the low frequency component is shown in Figure 2 (a) while that of the high frequency component is shown in Figure 2 (b). This histogram is for the decomposed image of Lena image. The histogram (a) is for LL component while the histogram (b) is for $\mathrm{HH}$ component. The histogram of the high frequency component looks similar to normal distribution and is mainly concentrated at the wavelet frequency component ranges from 0 to 10 wavelet frequency. On the other hand, Histogram of the LL component does not look like normal distribution.

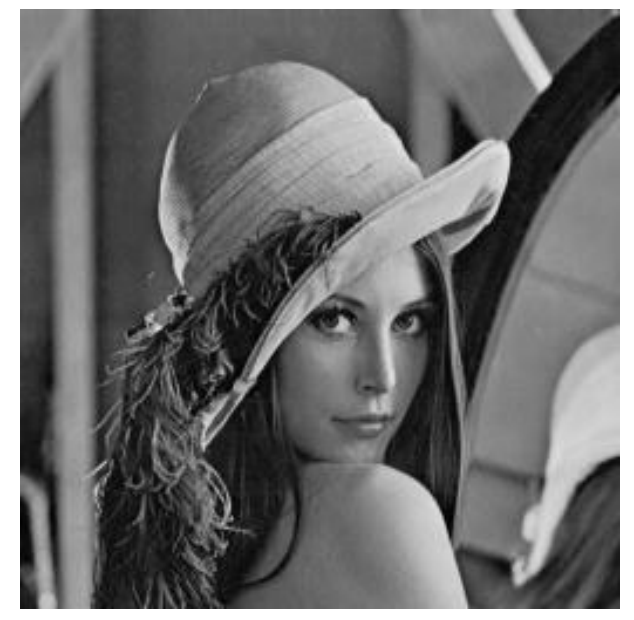

(a)Original image

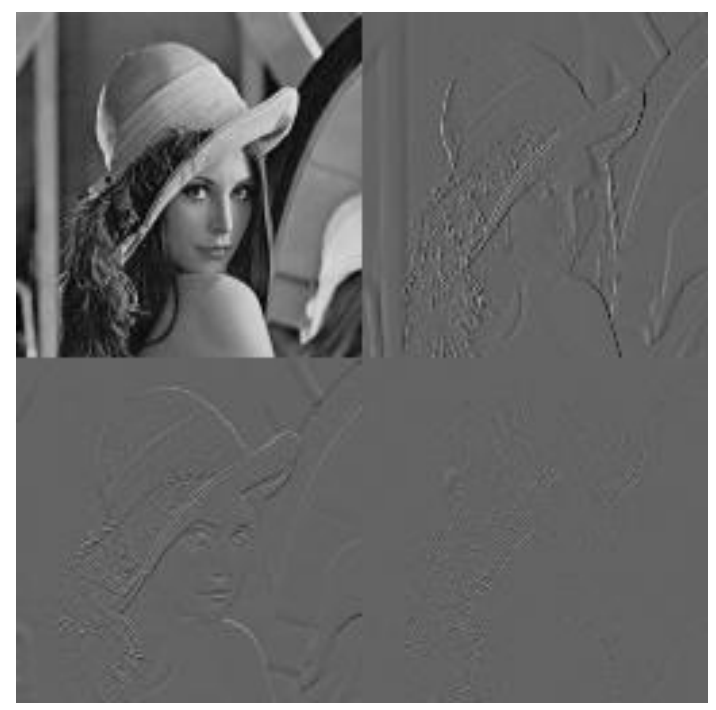

(b)Decomposed image

Figure 1 Example of original and decomposed images with DWT.

These histogram characteristics are common to original image and differ from each other depending on natures of the original images. Therefore, it is possible to separate original images using the difference of histograms of the decomposed image derived through DWT.

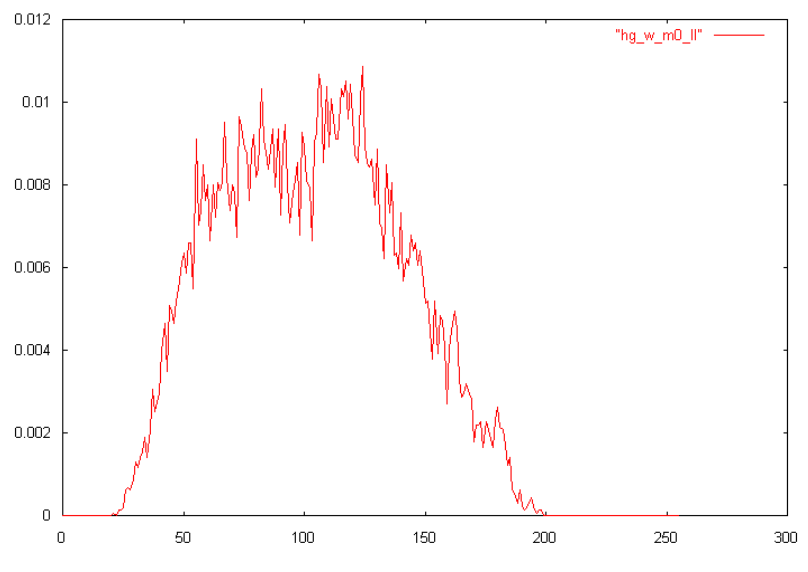

(a)Low Frequency Component 


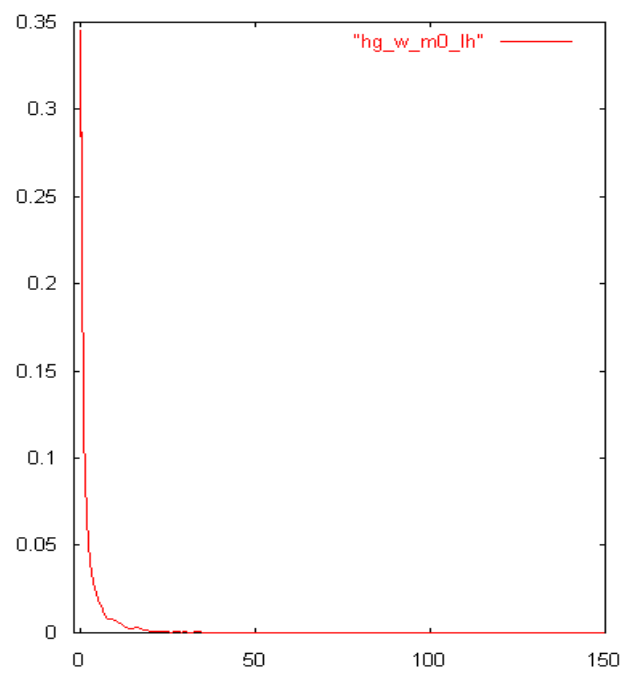

(b)High Frequency Component

Figure 2 Histograms of high and low frequency components of the decomposed image after DWT

\section{EXPERIMENTS}

\section{A. Original Imaes Used}

Figure 3 shows two original images, Lena, and Barbara in the same standard image database, SIDBA.

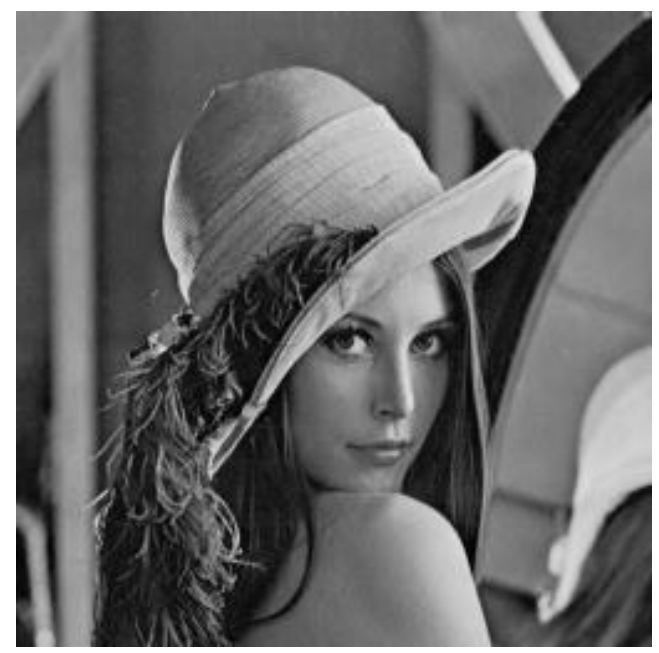

(a)Lena

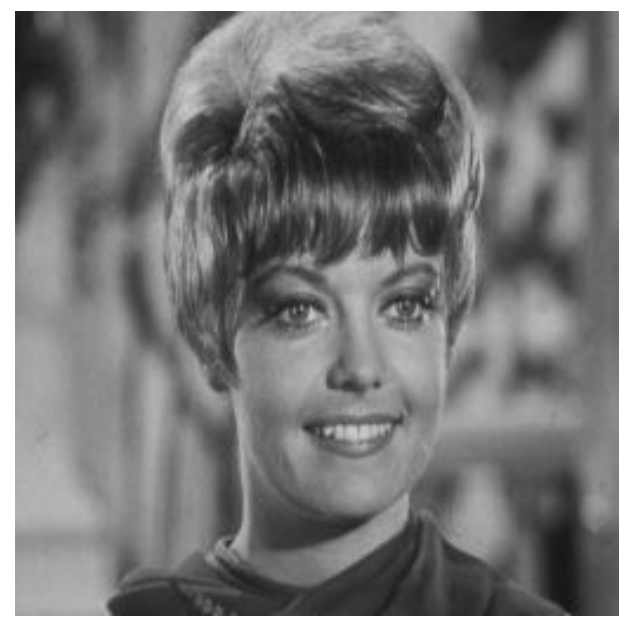

(b)Barbara

Figure 3 Two original images used for experiments

Also examples of mixed images are shown in Figure 4. Mixing ratios of Figure 4 (a) and (b) are different. Mixing ratio of Figure 4 (a) is $90 \%$ of Lena image and $10 \%$ of Barbara image while that of Figure 4 (b) is $10 \%$ of Lena image and $90 \%$ of Barbara image. Image As shown in Figure 4, image defects are found on the mixed, combined images.

Figure 5 shows DWT applied images of Figure 4 of combined images.

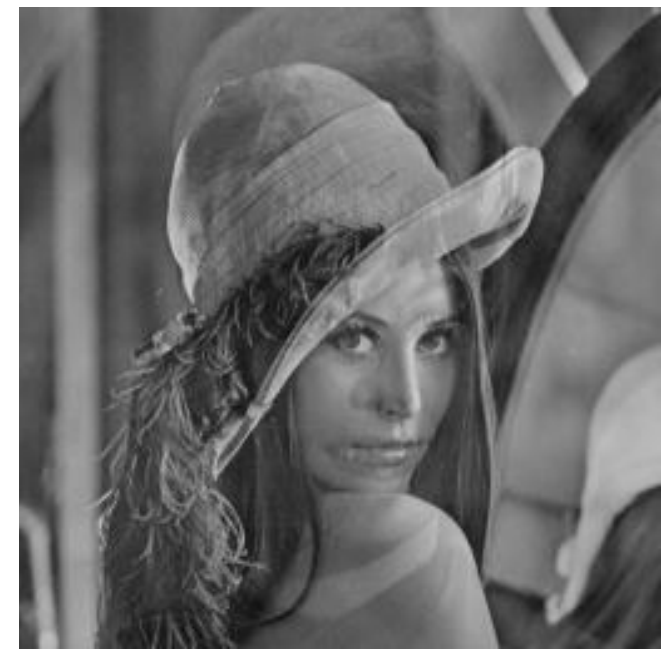

(a)Combined image with $90 \%$ of Lena and $10 \%$ of Barbara 


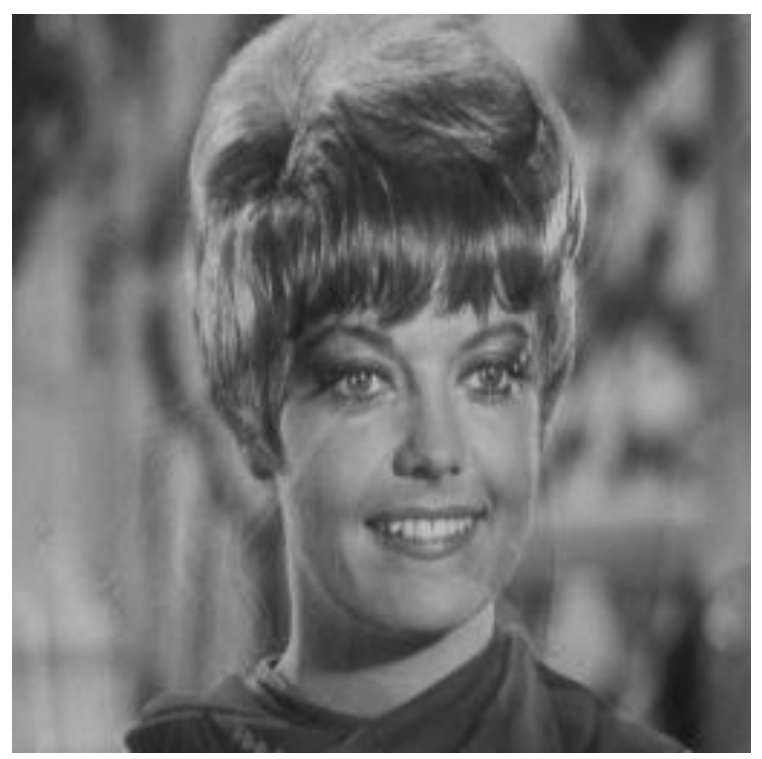

(b)Combined image with $90 \%$ of Barbara and $10 \%$ of Lena

Figure 4 Combined images with the different mixing ratios

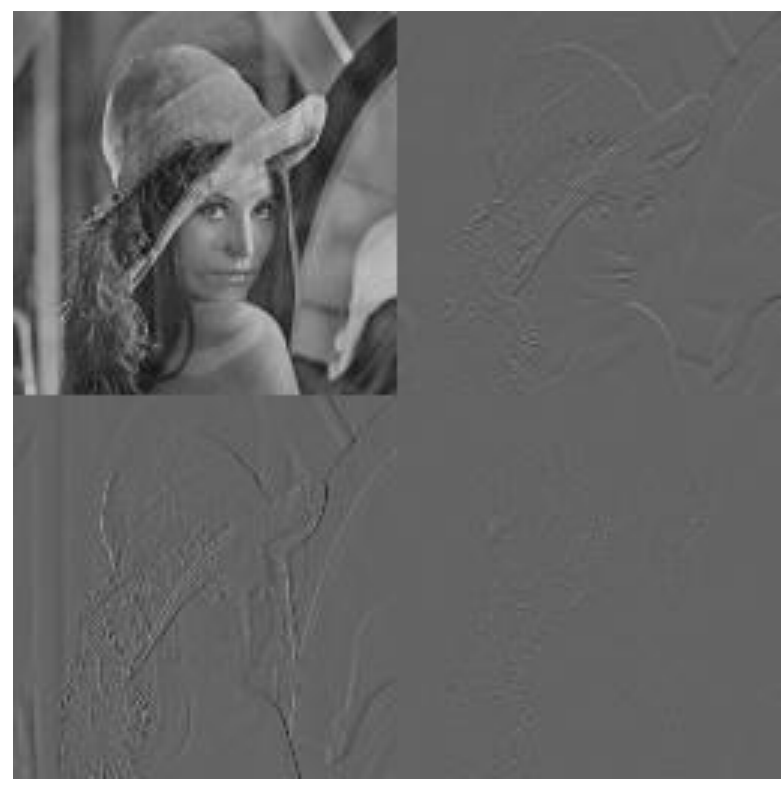

(a)DWT image of Figure 3 (a) of combined image

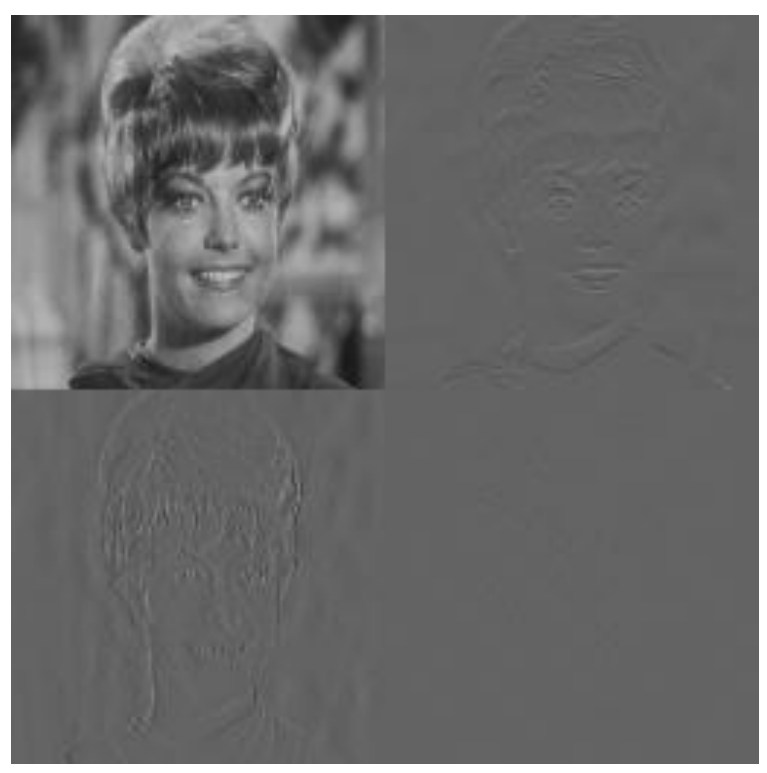

(b)DWT image of Figure 3 (b) of combined image

Figure 5 DWT images of Figure 4.

\section{B. Separated Images with the Different Mixing Ratios}

Mixed image between Lena and Barbara images with the different mixing ratios, $50 \%, 30 \%$, and $10 \%$ are created. Using the proposed method, separation of each original image is attempted. Resultant images are shown in Figure 6, 7, and 8 for the mixing ratios, $50 \%, 30 \%$, and $10 \%$, respectively. As shown in these Figures, image separation performance depends on the mixing ratio. It is difficult to separate image when the mixing ratio is around 50\%. Meanwhile, image separation performance is getting better in accordance with decreasing of the mixing ratio. In particular, the separated images for the mixing ratio of $10 \%$ are almost perfect, look extremely similar to the original images.

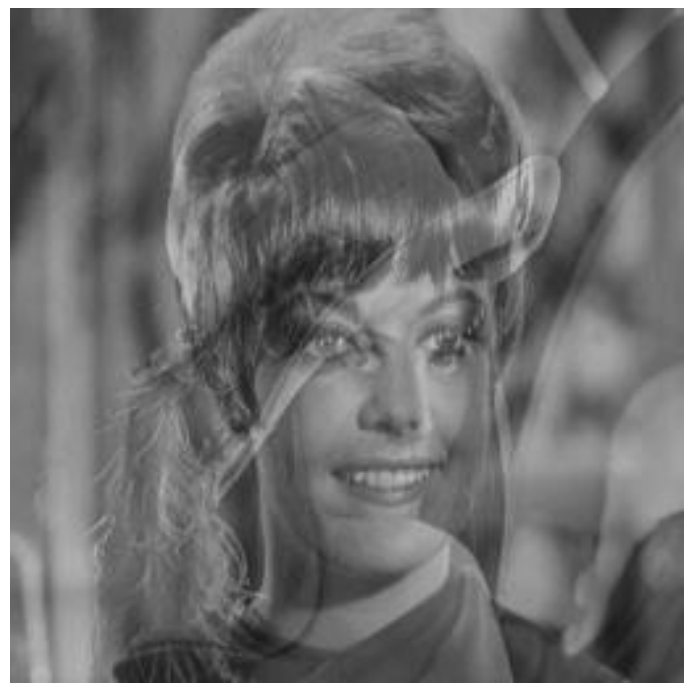

(a) 


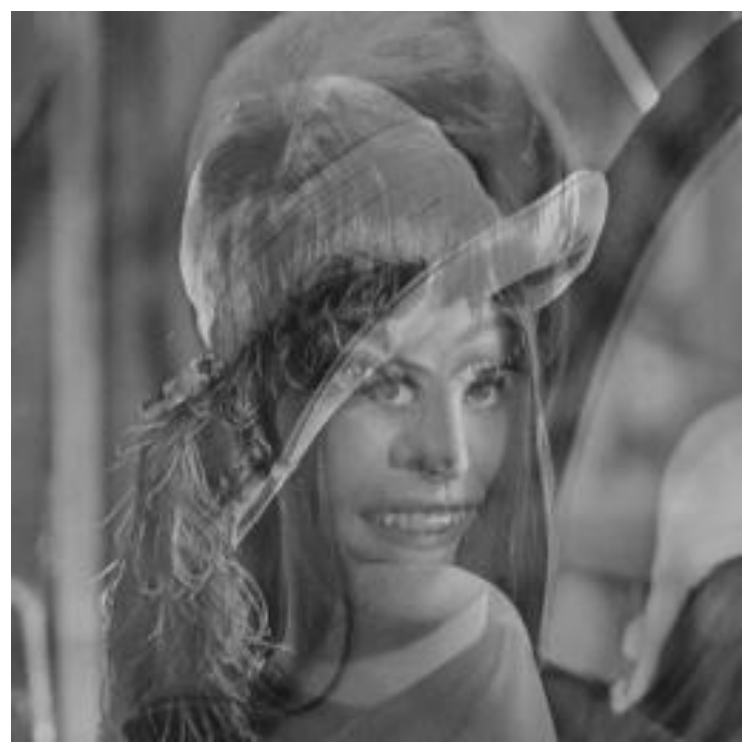

(b)

Figure 6 Separated images in the case of which mixing ratio is $50 \%$.

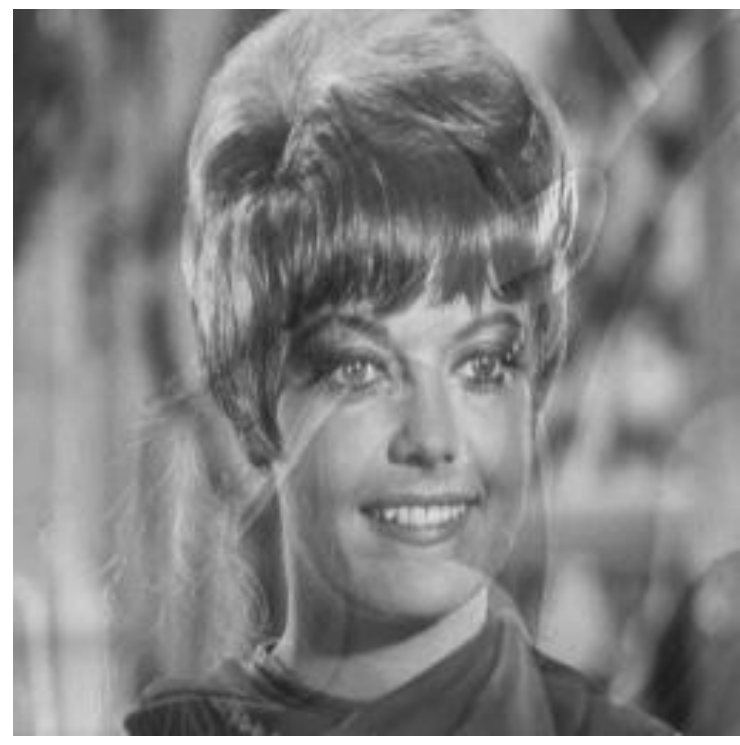

(a)

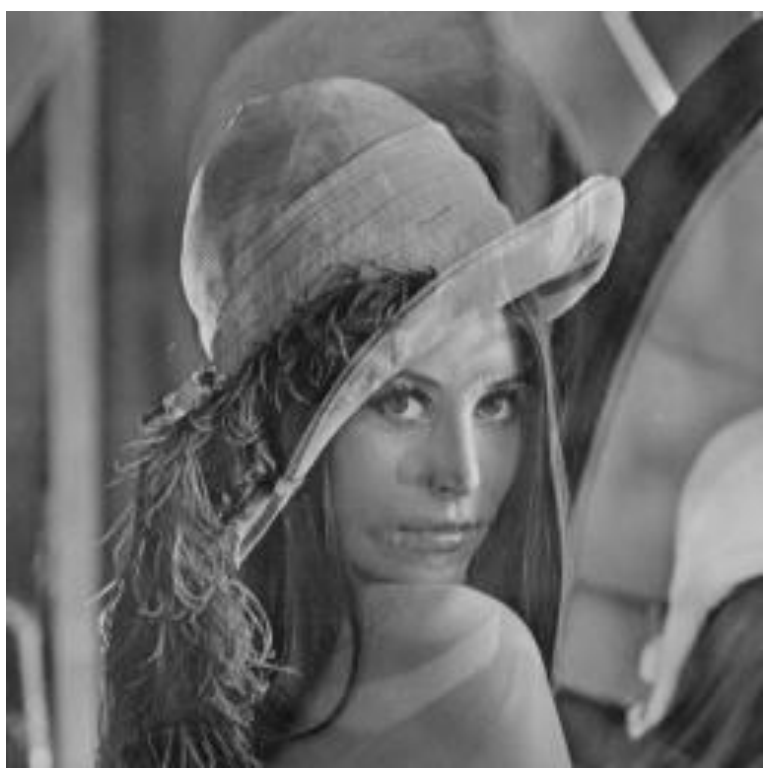

(b)

Figure 7 Separated images in the case of which mixing ratio is $30 \%$.

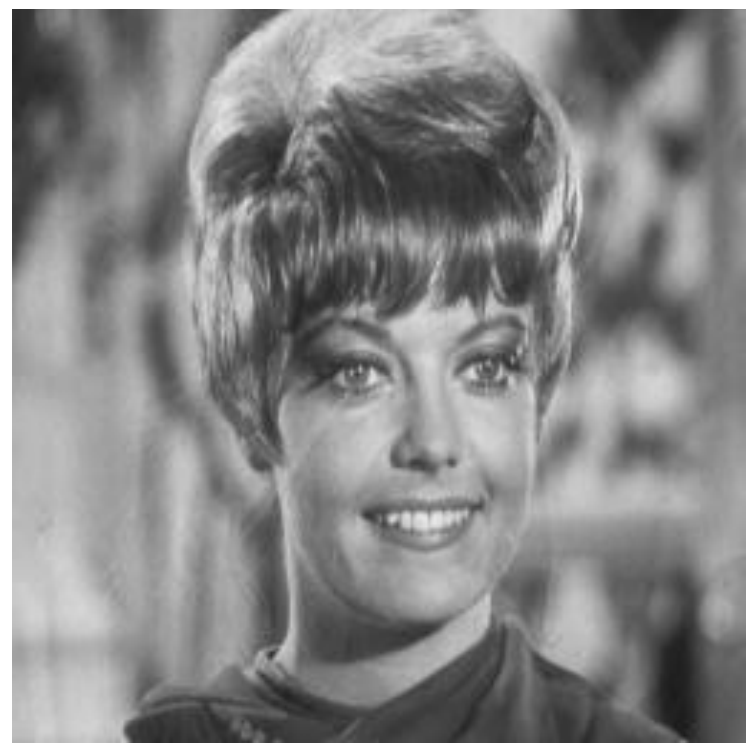

(a) 


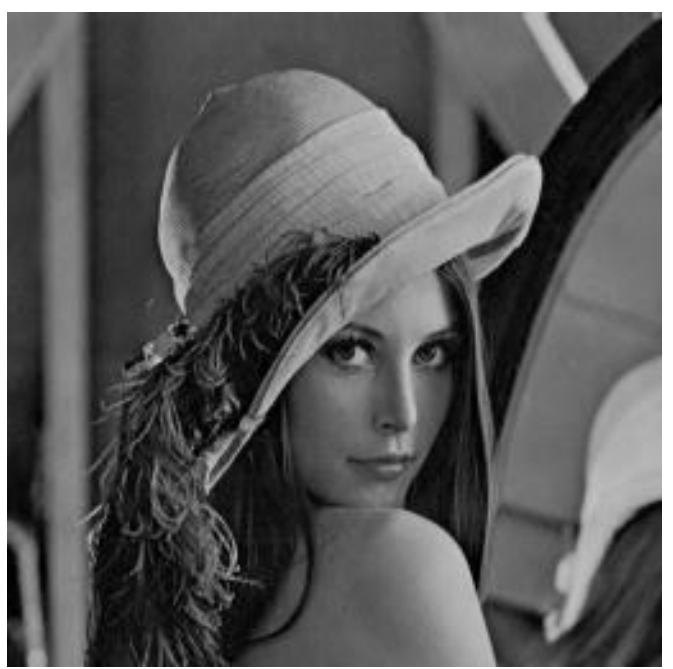

(b)

Figure 8 Separated images in the case of which mixing ratio is $10 \%$.

\section{CONCLUSION}

Method for image source separation based on Independent Component Analysis: ICA, Maximum Entropy Method: MEM, and Wavelet Based Method: WBM is proposed. Experimental results show that image separation can be done from the combined different images by using the proposed method with an acceptable residual error.

It is found that the image separation performance depends on the mixing ratio. It is difficult to separate image when the mixing ratio is around $50 \%$. Meanwhile, image separation performance is getting better in accordance with decreasing of the mixing ratio. In particular, the separated images for the mixing ratio of $10 \%$ are almost perfect, look extremely similar to the original images.

\section{ACKNOWLEDGMENT}

The author would like to thank Takeshi Yoshida for his effort to the experiments.

\section{REFERENCES}

[1] A. Hyvanrinen, J. Karhunen, E. Oja, Independent Component Analysis, John Wiley and Sons, 2001.

[2] H. Nomura, Y. Kaneda, N. Kojima, Near Field Types of Microphone Array, Journal of the Acoustical Society of Japan, 53, 2, 110-116, 1997.

[3] Y. Kaneda, Adaptive Microphone Array, Journal of the Institute of Electronics, Information and Communication Engineers, J71-B-II, 11, 742-748, 1992.

[4] M. Takagi and H. Shimoda Edt. K.Arai et al., Image Analysis Handbook, Tokyo-Daigaku-Shuppankai Pub. Co. Ltd., 1991.

[5] C.Jutten,J.Herault, Blind separation of sources, Part I: An adaptive algorithm based on neuron, Signal Processing, 24, 1-10, 1991.

[6] S. Amari, N. Murata, Indepent Component Analysis -A New Method for Multi-Variate Data Analysis, Science Pub. Co. Ltd., 2002.

[7] S. Morishita, S. Miyano Edt., K. Nijima, Extraction of hidden image signals by means of blind separation and wavelets, Kyoritsu Shuppan Pub. Co. Ltd., 201-206, 2000.

[8] K.Arai, Fundamental Theory on Wavelet Analysis, Morikita Shuppan Pub. Co. Ltd., 2000.

[9] K.Arai, Self Learning on Wavelet Analysis, Kindai-Kagakusha Shuppan Co. Ltd., 2003.

[10] K. Arai, T. Yoshida, Speaker separation based on blind separation method with wavelet transformations, Journal of the Visualization Society of Japan, 26, Suppl.1, 171-174, 2006.

[11] K.Arai, Blind separation, IJRRCS

\section{AUTHORS PROFILE}

Kohei Arai, He received BS, MS and PhD degrees in 1972, 1974 and 1982, respectively. He was with The Institute for Industrial Science and Technology of the University of Tokyo from April 1974 to December 1978 also was with National Space Development Agency of Japan from January, 1979 to March, 1990. During from 1985 to 1987, he was with Canada Centre for Remote Sensing as a Post Doctoral Fellow of National Science and Engineering Research Council of Canada.

He moved to Saga University as a Professor in Department of Information Science on April 1990. He was a councilor for the Aeronautics and Space related to the Technology Committee of the Ministry of Science and Technology during from 1998 to 2000 . He was a councilor of Saga University for 2002 and 2003. He also was an executive councilor for the Remote Sensing Society of Japan for 2003 to 2005. He is an Adjunct Professor of University of Arizona, USA since 1998. He also is Vice Chairman of the Commission "A" of ICSU/COSPAR since 2008. He wrote 30 books and published 322 journal papers. 